\title{
Microscale Transport Phenomena for Bio-Engineering Applications: Recent Advances
}

\author{
C. B. Sobhan ${ }^{1 *}$, Shijo Thomas ${ }^{1}$, and G. P. Peterson ${ }^{2}$ \\ ${ }^{1}$ National Institute of Technology Calicut, Kerala, India \\ ${ }^{2}$ Georgia Institute of Technology, Atlanta, Georgia, USA \\ Email: csobhan@nitc.ac.in
}

\begin{abstract}
The importance of microscale thermal energy transport has dramatically increased in the past several years and a number of significant investigations have been undertaken to better understand the fundamental phenomena that govern the behavior of energy transport at the microscale. While the original focus of this research was directed towards the thermal management of semiconductor devices and spacecraft thermal control, there has been an increased interest in the thermophysical phenomena occurring in biological systems, and bio-medical devices and applications. As a result a number of investigations have been reported, in which the thermal transport phenomena in microscale systems and passages are of significant importance. In this context, this following attempts to review the relevant literature on the theoretical and experimental investigations of microscale transport phenomena as specifically related to bioengineering and biomedical applications. Modeling methodologies, experimental studies, instrumentation techniques and research leading to the development of optimal bio-medical systems are reviewed and discussed.
\end{abstract}

Keywords: Microscale transport, bio-engineering, heat and fluid flow

\section{Introduction}

Thermal energy transport in physical domains of small dimensions has become increasingly important in the past two decades. Owing primarily to the scientific developments in the electronics and spacecraft thermal control arenas, which demanded miniaturization of the thermal control systems, research on microscale and nanoscale heat transfer grew tremendously. The applications of the new methodologies and approaches introduced were not limited to these areas and have expanded significantly in the past decade. Many of the innovations, experimental findings and analytical methods associated with microscale and nanoscale physical systems are fundamental in nature, and as a result, can be applied to a large number of applications in areas such as micro-fabrication methods, materials engineering, biological systems and bio-medical processes, apart from thermal management of microelectronics. Interestingly, the methodologies and measurement techniques developed to analyze heat transfer processes in microscale and nanoscale systems are also quite well suited for understanding the thermal phenomena in biological systems. They are also appropriate as tools in designing and optimizing processes and products related to biological systems. As a result, more recent research in microscale and nanoscale heat transfer has focused on biological systems and applications.

Analysis of heat transfer in domains with small dimensions often requires approaches different from those applied for conventional heat transfer problems [Sobhan and Peterson, 2008]. Fundamental transport coefficients, such as the thermal conductivity and viscosity, if defined using the continuum assumptions are of limited value in analyzing conduction or convection problems, as the sizes of the domains become very small. This results in what is known as the "size-effect" on the transport coefficients, due to the incomplete molecular interactions imposed by the domain boundaries. In convective heat transfer analysis, as the sizes of the passages become smaller and approach the molecular mean free path of the fluids in the passages, the usual assumption of no-slip boundary conditions in analyzing problems will have to be modified. As the dimensions become even smaller, the continuum assumption itself may prove to be insufficient, demanding molecular approaches in analyzing heat transfer problems. This situation may be encountered in the case of flow in nanochannels, under normal pressures, for the flow of liquids. These types of situations are not uncommon in biological 
systems, due to the small sizes of the passages, and the liquid and solid domains involved in the thermal interactions. Hence, special treatment of the problems related to the thermal phenomena becomes necessary to analyze many of the microscale and nanoscale heat transfer problems in biological systems. As direct instrumentation is almost impossible, and non-intrusive instrumentations such as optical instrumentation have their own limitations due to the inherent size limits imposed by the wavelengths of the electromagnetic radiations used, theoretical modeling and analyses often become essential to study such problems. Experimental research, developing and utilizing innovative techniques have also been undertaken, as reported in the literature. Theoretical and experimental approaches reported in the literature, aimed at analyzing microscale and nanoscale heat transfer problems related to biological systems are reviewed in this paper. Investigations have been conducted, in order to understand the physical mechanisms, as well as to implement the results in developing useful products and processes, which are relevant due to their application areas in bio-engineering. Some of the relevant research in the authors' laboratories is also discussed and reviewed.

One of the early articles which discussed the potentials and challenges of microscale heat transfer, and gave guidelines on how to approach problems that require special treatment due to size-affected phenomena in conduction and radiation, was published in 1994 (Tien and Chen, 1994). Focusing on conduction and radiative heat transfer, this paper identified different microscale heat transfer regimes, based on a comparison of the characteristic device dimensions to the heat carrier characteristic lengths, reviewed the methods of analysis, and discussed the difficulties encountered in the analysis and experimental research, prevalent at the time of its publication. The topics addressed were of relevance to generic applications. Microscale convective heat transport has been reviewed extensively in later publications, discussing the prospects as well as the challenges in modeling, and instrumentation, as well as bringing out the risks in making conclusions based on external observations and intuitive predictions (Sobhan and Garimella, 2001; Sobhan and Peterson, 2008). However, most of the work reviewed was focused on engineering applications such as those in thermal management of microelectronics, and little attention was devoted to the advances in the area of biological and bio-engineering applications.

Though the fundamental mechanisms of microscale heat transfer could be similar in engineering and biological systems, the nature of the physical domains, as well as the levels of sophistication required in the analysis and experimentation can be much different.

A consolidated article on a US National Science Foundation-sponsored workshop entitled "Frontiers in Transport Phenomena Research and Education: Energy Systems, Biological Systems, Security, Information Technology, and Nanotechnology" held in May of 2007 at the University of Connecticut has been published by Bergman et al. (2008). The importance of heat transfer research focused on biomedical and bio-engineering applications has been discussed in the paper, indicating the biotechnology challenges would benefit from the contributions of thermal science research. The topics pointed out in this paper included characterization and manipulation of physical and transport properties of biological materials including biomolecules, cells, tissues and arterial walls, modeling of transport phenomena in biological systems, developing engineered biosensors and actuation methods, and developing system approaches to study complex biological processes. The discussions presented specific guidelines for future research.

It would be interesting and useful to look into the current state of the art in thermal science research, relevant to bio-engineering applications, in order to get a picture of the directions of growth, as well as to understand the challenges involved in this area. As a part of this venture, this article focuses on research related to microscale thermal transport phenomena in biological systems and applications, and discusses methodologies and findings, by categorizing and reviewing some of the recent advances.

\section{Theoretical Analysis of Transport Phenomena: Conventional Models}

Modeling of heat transfer problems related to biological systems and bio-engineering applications using conventional continuum methods and their solution using analytical and numerical solution techniques have been the object of a large number of investigations. Macroscale bio-heat transfer have been helpful in understanding processes, and applying the findings in innovative designs, and modified processes. Recently, Wang and Fan (2011) have developed and discussed macroscale thermal models for biological tissues by the continuum mixture theory, which scales down the physical approach from the global scale, 
and the porous media theory which scales up from the microscale by volume averaging of microscale properties. However, it is well known that essentially the fundamental thermal transport processes in biological systems are microscale in nature, which may be size-affected, and simplifying assumptions might not provide reliable predictions from size-averaged theoretical models. In order to obtain a clear picture of the physical phenomena of thermal energy transport in biological systems, a microscale or nanoscale analysis would be required. Further, with the advent of Micro/Nano Electromechanical Systems (MEMS/NEMS), the possibilities of applications of engineered systems in bio-technology have been extended to miniaturized engineering systems and devices, which require microscale and nanoscale analysis of the processes, in order to apply the prediction methods to bio-engineering designs.

Bischoff and Rubinsky (1993) developed a set of heat and mass transfer equations to predict vascular and intracellular ice formation during freezing in liver tissue. The intracellular ice formation was predicted using a probability integral, which depended on the volume of the cell compartment, temperature, and time. Finite Difference method was used to make transient prediction of the temperature distribution in the tissue and the freezing interfaces. Bischoff (2006) has discussed in his interesting publication, micro and nanoscale bio-heat transfer problems, mainly focusing on thermal therapies, and mentioning also some cryo-preservation applications. Molecular (nano), cellular (micro) and tissue level (nano, micro and macroscopic) interactions of probes, thermal sources and sinks with biological systems have been mentioned in this paper. Pertaining to microscale (cellular) biophysics, mathematical models of cryothermic events, describing cellular dehydration and intracellular ice formation were discussed. In the case of hyperthermia and thermal therapy, time-dependent models for damage accumulation have been described. The paper also reviewed in-vitro and in-vivo measurement and imaging of cryothermic and hyperthermic injuries.

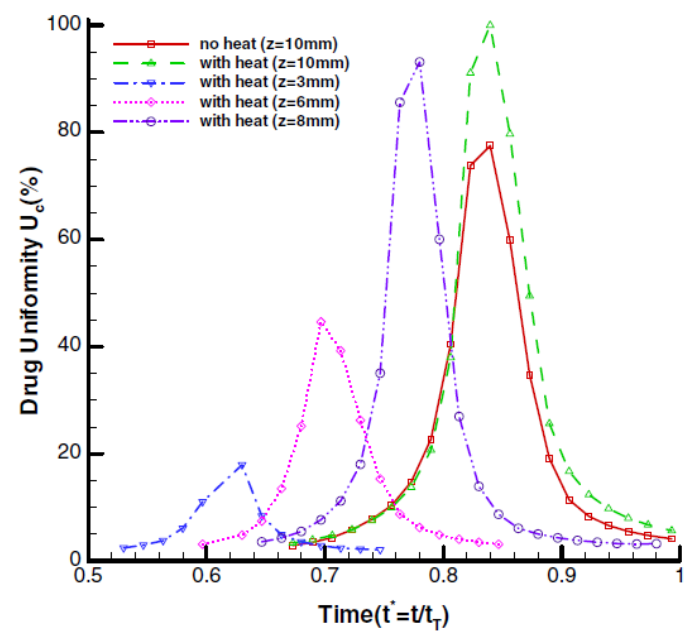

Figure 1. Comparison of drug concentration uniformity for different thermal boundary conditions in outlet planes and/or different axial stations, for a particle diameter of $10 \mathrm{~nm}$. From Kleinstreuer et al. (2008).

An interesting computational analysis of the microfluidics of nano-drug delivery through microchannels has been presented by Kleinstreuer et al. (2008). The investigation involved computational analysis of the propagation of drug nanoparticle suspensions in microchannels. The discussions were focused on the optimal delivery of drug nanoparticles in an aqueous suspension (nanofluid). Uniform particle concentration and uniform temperature of mixing are the primary concerns in such an application, where the dilute suspensions are conveyed to living cells in a well, through microchannels, from a plenum chamber. Computational solutions of the tree-dimensional time dependent governing equations, namely the momentum, energy and concentration equations were obtained using a finite volume procedure, while utilizing correlations for nanofluid properties. The model was validated against theoretical predictions for friction factor in rectangular channels. The major results presented in this paper are those of concentration distributions in the nanofluid, such as the ones shown in Fig. 1 and 
Fig. 2. The nanoparticle uniformity was quantified using an index defined as

$$
U_{c}=\left(A_{v} / A_{g}\right) \times 100 \%
$$

where, $\mathrm{A}_{\mathrm{v}}$ is the area where the particle concentration was equal or larger than $90 \%$ of the local maximum concentration, and $\mathrm{A}_{\mathrm{g}}$ is the channel exit area.

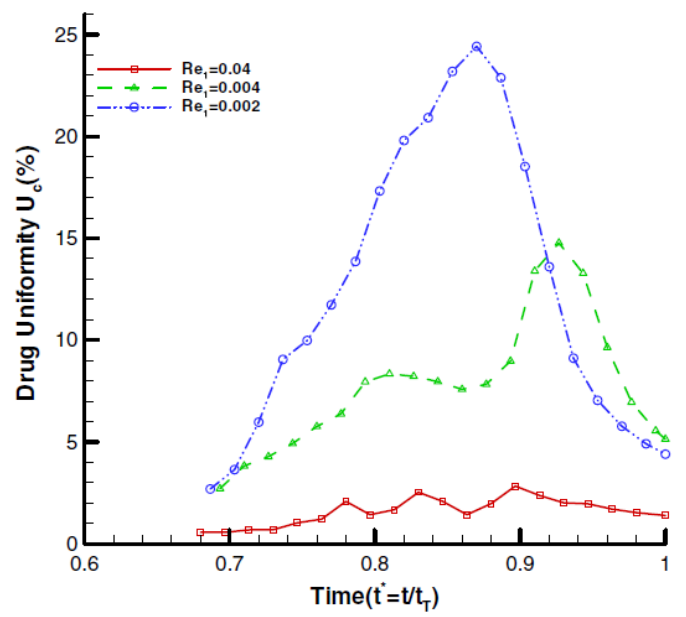

Figure 2. Comparison of drug concentration uniformity in the outlet plane at an axial location of $10 \mathrm{~mm}$, for different Reynolds numbers. Particle diameter is 500 nm. From Kleinstreuer et al. (2008).

Kathawate and Acharya (2008) performed computational modeling of the drug delivery process with different vitreous substitutes, in the vitreous chamber of the eye. The analysis dealt with drug distribution within the eye following intra-vitreal injection of drug for treatment of vitreo-retinal diseases. It was concluded that the concentration distribution depended on the properties of the vitreous substitute, the diffusion coefficient of the drug and the permeability of the retinal surface. For drugs with low diffusion coefficients, along with low viscosity vitreous fluids, convection was found to play an important role, which may produce toxically high drug concentrations on the retina. The work utilized numerical solution of the continuity, momentum and species transport equations, within the vitreous chamber of the eye, using the FLUENT software package.

Reversible electroporation is a method by which cell membranes can be made permeable, reversibly, by exposing them to strong electric pulses of microsecond rates. The approach is used to introduce molecules and genes into human cells. Granot and Rubinsky (2008) introduced a multiscale mathematical model to analyze mass transfer into cells during reversible electroporation of tissues. The model combined a macroscopic model for the electrical field, a cell-scale model for electroporation, and a macroscopic model for tissue-level mass transport. The applicability of the model was illustrated for a situation typical for electrochemotherapy for cancer, in predicting the concentration distributions of the drug using FEM solution. Analysis of the temperature fields during drug delivery in tissue cells with reversible electroporation has also been presented by Davalos and Rubinsky (2008). In order to obtain the temperature distribution, the mathematical model involved FEM solution of the modified form of Pennes bio-heat transfer equation (Pennes, 1948) in the following form:

$$
\nabla \cdot(k \nabla T)-w_{b} c_{b} T+q^{\prime \prime \prime}+\delta|\nabla \phi|^{2}=p c_{p} \frac{\partial T}{\partial t}
$$

Devireddy et al. (2002) performed numerical investigations of freezing in biological tissues. The effects of microscale mass transport and phase change on freezing were studied. The heat transfer problem was formulated as a one-dimensional model, solved using finite volume method, and the latent heat release was determined using a combined thermal- biophysical approach. The results suggested that the microscale biophysical processes in the biological tissues during freezing do not significantly limit the rate at which phase change occurs. Kandra and Devireddy (2008) presented numerical solution of a two dimensional unsteady conduction model to predict the time-depended temperature history of biological 
cells during ice-nucleation in suspension. Computations were presented assuming single, duel, and four cells in suspension. The results were analyzed to predict the magnitude and time response of perturbations to the local temperature field after ice nucleates within a cell. The analysis was aimed at estimating effective sampling rates of microscale thermocouples to measure temperatures during intracellular ice formation. A typical result of the solution is shown in Fig. 3.

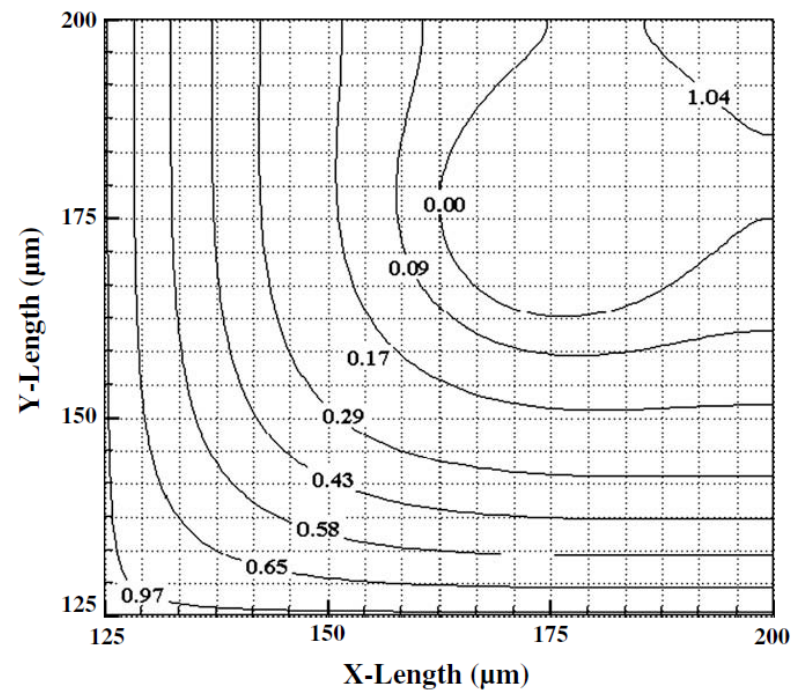

Figure 3. Typical thermal distortion contours in a cell at time $=1.96 \mathrm{~s}$, after the simultaneous nucleation of ice within four cells. From Kandra and Devireddy (2008).

$\mathrm{Xu}$ et al. (2010) described multiscale approaches for modelling cell and tissue cryopreservation. The discussions included aspects of heat transfer at macroscale level, crystallization, cell volume change and mass transport across cell membranes at microscale level. Physical and mathematical models were dealt with extensively, with case studies presented.

Theoretical modeling has been applied to predict the behavior of specific biological systems and processes, where models have been numerically solved. These studies pertain to a wide spectrum of problems, and are diverse in applications.

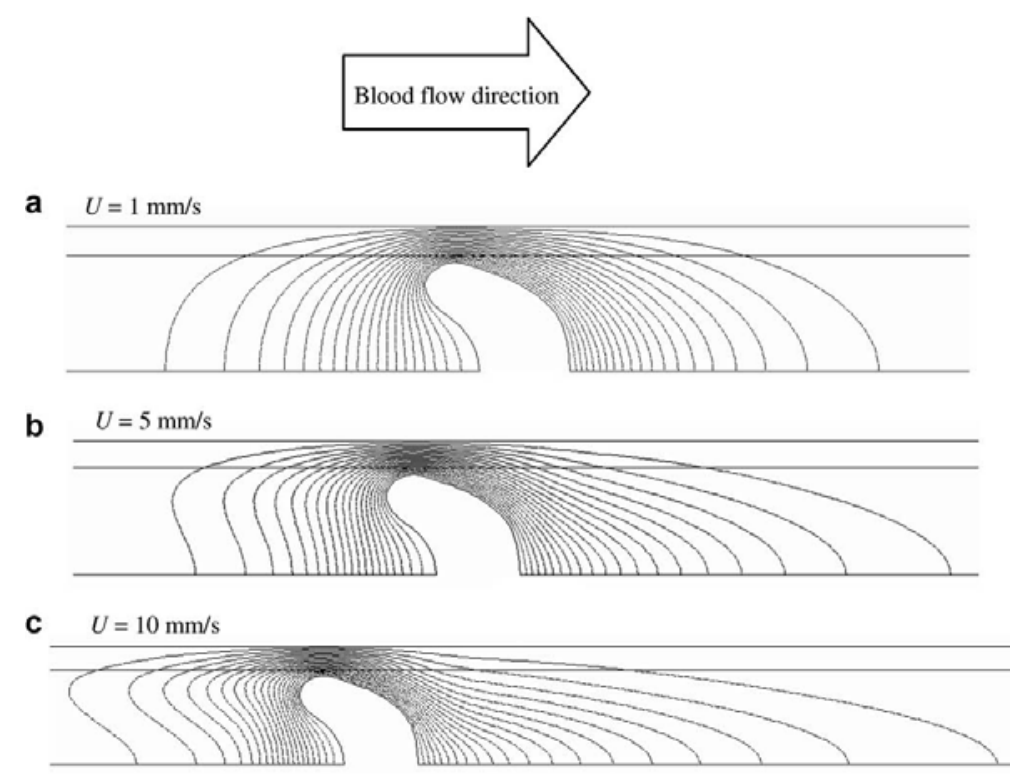

Figure 4. Isobaric lines for a single $\mathrm{RBC}(\mathrm{Ht}=3.09 \%)$ flowing in the capillary with (a) $\mathrm{U}=1 \mathrm{~mm} / \mathrm{s},(\mathrm{b}) \mathrm{U}=5$ $\mathrm{mm} / \mathrm{s}$ and (c) $\mathrm{U}=10 \mathrm{~mm} / \mathrm{s}$. From Merrikh and Lage (2008). 
Fleming Glass et al. (2008) presented optimization study of a microfluidic device, designed for extraction of Dimethyl Sulfoxide (DMSO) from a cryopreserved cell suspension. The method was based on diffusion, and used a two-stream (a cell suspension stream and a parallel wash stream) microfluidic device. Numerical computations leading to optimization of the geometry of the device as well as the operating conditions are presented in this paper.

$\mathrm{Xi}$ and Longest (2008) performed computational studies to predict aerosol deposition in the nasal cavity. The aerosol particles considered were sub-micron in size. The modeling was based on a drift-flux approach. The geometry of the nasal cavity geometry was developed based on MRI data. A correlation for mass transfer and deposition of particles in the nasal airways was proposed, which can be applied for sub-micrometer aerosols.

Merrikh and Lage (2008) studied plasma microcirculation in blood capillaries. The effects of plasma microcirculation speed and red blood cell (RBC) shape on carbon monoxide exchange in alveolar (lung) capillaries were analyzed using relevant fluid dynamic and diffusion equations, solved numerically using Finite Volume method. Blood was modeled as a two-constituent substance, consisting of the blood plasma, assumed to be a homogeneous Newtonian fluid, and the red blood cells (RBC) modeled as discrete, suspended solid particles in the plasma medium. The effects of the shape of the RBC shape and the velocity of blood on the alveolar diffusion process were predicted, and represented using the variations in streamlines and isobaric lines (typical results are reproduced in Fig. 4), as well as quantified using a capillary diffusing capacity of the lung.

A novel cancer treatment method utilizes albumin coated liquid droplets of a perfluorocarbon, as small as 6 microns in diameter, injected into the blood stream, which subsequently vaporize selectively to produce large vapor bubbles. Motivated by this physical system, a mathematical model has been presented by Eshpuniyani et al. (2008), where the system of governing equations for Stokes' flow with a bubble sliding along one of the walls of a $2 \mathrm{D}$ channel has been solved using the boundary element method. Pressure distributions and stress profiles along the channel walls were obtained. The obstruction of flow through the channel due to the bubble was also studied.

Santos et al (2008) modeled heat transfer in large blood vessels during radiofrequency tumor ablation, to obtain the tissue temperature, and the effect of the assumption of a variable heat transfer coefficient in it. A time-dependent model with the heat transfer coefficient as a function of the temperature distribution at the wall of the blood vessel was used. Finite-element method was utilized in the solution. It was concluded that for longer time tumor ablation procedures of the order of 5 minutes, a constant heat transfer coefficient assumption was sufficient to obtain precise predictions.

Axons transmit electrical signals from neurons to cells. Kunzetsov and Hooman (2008) have modeled the intracellular transport in axons. The paper suggested a model for traffic jams caused by Irregularities in intracellular traffic in axons, due to mutations of molecular motors, producing various neurodegenerative diseases. The model was based on the motion of intracellular particles under the combined action of diffusion and motor-driven transport. The solution was obtained using the Finite Difference method.

Understanding non-Newtonian blood flow in curved channels with circular or elliptical cross sections is important in bio-mechanics. A theoretical analysis of creeping viscoelastic flow has been performed by Norouzi et al. (2013), aiming at the convective heat transfer in a curved circular pipe. The flow and heat transfer were assumed to be fully-developed, with a constant heat flux at the walls. The effects of normal stress differences on heat transfer were investigated. The investigation gives useful information on arterial bio-fluid dynamics.

An interesting theoretical study of the vapor-liquid phase interaction in flare flashing sprays used in dermatolegic cooling was presented by $\mathrm{Vu}$ et al. (2008), in which flashing of a high superheat fluid flowing through micro tube nozzles, resembling medical devices, was investigated. A one-dimensional, semi-empirical model of refrigerant flow through capillary tubes was used in this analysis, in which solutions of governing conservation equations were obtained using numerical methods. Experimental results using flash lamp photography were also presented. Another interesting theoretical investigation on a specific problem has been reported by Chen et al. (2008), on transport of water and cryoprotectants through dendritic cell membrane. A biophysical model was used for the analysis to determine the transport properties of the cell membrane, and the cell volume changes under various extracellular conditions (in mouse dendritic membrane) were also observed using a video camera. The 
work has relevance in cryopreservation of dendritic cells, which is of immense value in immunotherapy treatment.

Investigations on heating due to magnetic fluids (biocompatible superparamagnetic nanoparticle suspensions), in magnetic fluid hyperthermia of tumor with blood profusion, have been reported. The effects of magnetic nanoparticle properties were studied. Finite difference schemes (Kappyoor et al., 2010) and analytical method (Liangruksa et al., 2011) have been utilized to obtain the solutions of the governing equations.

In most biological detections specimens at low concentrations are mixed, which may lead to exothermic reactions, and hence need a microfluidic thermal management system (MTS). Lee (2013) developed a refrigerant based on iron-ferrite magnetic particles for a heat pipe based MTS. The evaporator section was disk-shaped porous wick with good capillary characteristics and high effective thermal conductivity. The use of a magnetic microfluid in the cooling section showed an enhancement in cooling performance upto $25 \%$.

Sinha and Shit (2015) conducted a theoretical study on the electromagnetohydrodynamic flow of blood through a capillary subjected to constant heat flux. In electrohydrodynamic flows of conducting fluids electric field applied can result in joule heating resulting in temperature rise and creation of temperature gradients thus culminating in loss of resolution during analysis. Infrared/ultrasonic radiations used in treatment of muscle spasms, breast tumors, etc. are found to affect blood flow. The present study showed that magnetic field can be used to regulate blood flow particularly during surgery. As the magnitude of joule heating increases the spatial distribution of blood temperature uniformly increases and decreases temperature gradient at capillary walls thus reducing the heat transfer characteristics.

\section{Laser Heating of Tissues}

Surgery, chemotherapy and radiation are the most popular treatment methods used for cancer. Off-late hyperthermia treatments like radiofrequency ablation, microwave, laser, and focused ultrasound are used as the modern therapeutic methods used for destroying tumors through safe heating of biological tissues. In thermal therapy method using focused laser beam a pre-determined volume of heat is delivered to the affected tumor region. Accurate prediction of the temperature field and extend of damage to the tissue is important in such exercise. Modeling of biological tissues with blood perfusion presents a challenging problem, as it often needs non-Fourier models to describe the conduction phenomenon, and because of the non-Newtonian behavior of blood during its perfusion through the tissues. A number of investigations have been reported on laser heating of skin and blood-perfused tissues. Arkin, et al. (1994) have reviewed the developments in modeling heat transfer in blood perfused tissues. Liu et al (1999) presented discussions on thermal wave aspects of the theoretical analysis and evaluation of burn of skin subjected to instantaneous heating. Comparative study of the Pennes equation and the newly developed Thermal Wave Model of Bio-heat Transfer (TWMBT) has been performed in this paper. Interesting results for temperature prediction in the skin using theoretical models were discussed, as shown in Fig. 5. Subsequent studies have also applied both the Pennes model and TWMBT to investigate the thermal behavior of tissues during external laser heating. Liu (2000) has also presented a survey on the wave-like behavior of heat transfer in living tissues.

Katsidis (2002) studied the effect of lasers and light absorption on human skin analytically, using the Pennes bio-heat transfer (PBTE) model. The thermal interaction of short-pulse laser with skin tissue cylinder was numerically studied by Jiao and Guo (2009). They observed that the focused beam can penetrate a greater depth and generate higher temperature rise at the target area, thus reducing the possibility of thermal damage to the surrounding healthy tissue. Banerjee et al. (2005) analyzed temperature distributions in different materials caused by short-pulse laser irradiation with both Fourier and non-Fourier heat conduction models. They compared the measured temperatures with those obtained by the Fourier, as well as non-Fourier model and observed that the non-Fourier results agreed better with the experimental data. Kim and Guo (2007) presented a combined radiation and conduction model to simulate the multi-time-scale heat transfer in turbid tissues exposed to short-pulse irradiation. They found that the maximum local temperatures obtained from a hyperbolic model are greater than those predicted by the parabolic model. Jaunich et al. (2008) solved the bio-heat transfer equation for 
short pulse laser irradiation on body tissues. They validated their numerically generated results by examining the experimental data, and results showed that the experimentally measured temperature distributions agreed better with the predictions made through the hyperbolic heat conduction model. Molina et al. (2008) presented an analytical solution for the hyperbolic heat conduction model in cylindrical coordinates for the following typical samples of heat-treated biological tissues: heating of the cornea for refractive surgery, cardiac ablation for eliminating arrhythmias, and hepatic ablation for destroying tumors. They applied a two layer model to describe the heat conduction process in the skin tissue. A dynamic photothermal model of $\mathrm{CO}_{2}$ laser tissue ablation was developed by Zhang et al. (2008). They solved the PBTE model numerically using the finite difference method to predict the temperature history and laser energy field deposition. Coupled photon and heat transport simulation in biological tissue for laser therapy was performed by Sakurai et al. (2009). They employed the finite volume scheme for solving the governing equations and observed that the temperature distribution inside the tissue changed slightly with the optical properties. Their results indicated that the optical properties of biological tissues are quite sensitive to the temperature characteristics. Zhou et al.(2009) numerically studied the thermal damage to biological tissues caused by laser irradiation through the dual-phase-lag (DPL) bio-heat transfer model using a finite volume scheme. They compared their results with the Fourier, as well as thermal wave models, and observed that their approach predicted significantly different temperatures and thermal damages in tissues. A two-dimensional axisymmetric DPL model was considered, and solved numerically for the laser heating of living tissue by Zhou et al. (2009). The numerical data were compared with analytical results to validate the numerical scheme. It was found that when the heating spot became equal to the thickness of the cylinder, the numerical data agreed very well with the analytical results. A study on the photothermal mechanism of laser-skin interaction has been presented by Guan et al. (2011). Ozen et al. (2011) applied the TWMBT model and the Pennes equation to predict the burn injury of skin tissue exposed to microwaves. They solved the resultant governing equations numerically, and observed that the TWMBT predicts a lower temperature rise than the PBTE model.

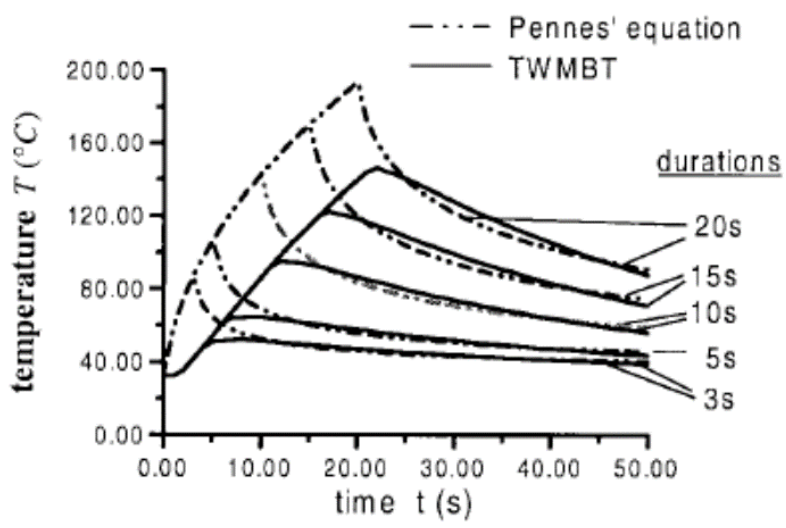

Figure 5. Temperature prediction at the interface between epidermis and dermis for different heating durations. Reproduced with permission from Liu et al. (2002).

Sarkar et al.(2015) provides an analytical solution using Pennes equation for a two dimensional cancerous skin tissue with five layers. The effect of heat generation during thermal therapy on the skin is analysed by deriving the temperature distribution on individual skin layers. The analytical solution was found to be within $0.04 \%$ of the FEM results. The maximum rise in temperature was observed in the region where the tumour is present. It was also found that with decrease in tumour size the temperature distribution widens and reduces the maximum temperature rise.

Grabski et al.(2016) models the time dependent blood perfusion in biological tissues using the Pennes equation which is formulated as an inverse problem and solved using the method of fundamental solutions.

Ma et al.(2016) modeled brain as consisting of two layers, scalp and brain matter, the thermal response of which was modeled using the Pennes equation. The solution of the temperature distribution was obtained using the Laplace transform method. 
Wang et al.(2015) proposes a mathematical model for predicting the temperature distribution in the achilles tendon of human subjected to radiofrequency ablation. The biological tissue is modeled as a porous structure with blood perfusion, the blood flow in the tumor region being modeled using the Darcy flow model. The ablation probe is modeled as a heating source. It was found that the mechanical properties of tissues like Young's modulus and skin tissue stiffness got reduced as temperature rises due to heating.

Wang et al.(2015) investigated the combined effect of cryosurgery and hyperthermia for cancer treatment using coupled heat transfer and fluid flow models in a 3D space. The 3D vascular network in the tissues was modeled using fractal theory based on MRI images. The heat transfer in the interstitial spaces of biological tissue is modeled using the Pennes equation and the flow and heat transfer in vascular network is modeled as the Navier Stokes equations (continuity, momentum and energy).

The usage of laser for therapeutic and diagnostic purposes has increased the risk of accidental exposure of human eye to lasers and causing damage. Heussner et al.(2014) developed a three dimensional thermodynamic model of the human eye which takes into account the effect of irradiation on sclera, retina, choroid and eye lid. The effect of vertical blood flow in the choroid is also considered as it is important in long exposures. The model can predict the temperature rise and hazards caused by irradiation. The results were compared with measurements in rabbit.

Wessapan and Rattanadecho (2013) analysed the effect of electromagnetic radiation exposure and frequency on human eye to quantify the Specific Absorption Rate (SAR) and temperature distribution. It was found that the cornea was subjected to the highest SAR at frequencies of 900 and $1800 \mathrm{MHz}$. The highest temperature was in the anterior chamber and vitreous at frequencies of 900 and $1800 \mathrm{Mz}$, respectively.

\section{Non-Conventional Theoretical Models}

In some of the physical problems related to biological heat transfer, analysis based on Non- Fourier models are required, due to the special thermal behavior of biological cells and tissues. Further, owing to the size limited domains and size affected phenomena, often encountered in microscale and nanoscale transport, conventional models based on continuum hypothesis and solutions using usual numerical methods also sometimes prove to be insufficient in analyzing biological systems. In such cases, modified models, and discrete computation methods are applied. The parabolic heat diffusion theory based on Fourier's law breaks down in the case of heating in biological tissues owing to their non-homogeneous material structure and high power with short durations, occasionally subjected to cryogenic temperatures. The Fourier's law assumes that the electrons and atomic lattice attains thermal equilibrium instantaneously and the thermal equilibrium propagates at high speed through the medium, which fails in the case of biological tissues. The heat transfer analysis of biological tissues has been performed using the Pennes Model of bioheat transfer equation (PMBTE), Thermal Wave Model of bioheat transfer equation (TWMBTE) and Dual Phase Lagging model for heat conduction. Pennes model based on macroscopic heat diffusion theory fails to take into account the finite speed of thermal wave propagation. The Thermal Wave model, at times, generates solutions like negative thermal energies and negative entropies which are physically impossible, due to the inability to consider relaxation times between electrons and atomic lattice. There have been interesting investigations reported in the literature, where special modeling and solution techniques have been applied. Some of them are reviewed here.

Zhang (2009) investigated on non-equilibrium heat transfer in living biological tissues. Dual-phase lag bioheat equations were obtained, with the temperature of blood or tissue as the sole unknown temperature, based on the non-equilibrium model. In the dual-phase model, the phase lag times were expressed in terms of the properties of blood and tissue, the interphase convective heat transfer coefficient and blood perfusion rate. The model was found to be superior to the dual-phase lag bioheat equation normally obtained as a modification of the classical Pennes bioheat equation. From the model, it was found that the phase lag times for heat flux and temperature gradient for the living tissue are very close to each other.

Askarizadeh and Ahmadikai compares the PMBTE, TWMBTE and DPL models of bioheat transfer on a skin tissue subjected to instantaneous heating, considering the effects of blood perfusion and 
metabolic heat generation. The analytical solution was arrived at using the Laplace Transform (LT) technique coupled with inversion theorem and separation of variables method. It was found that at the conditions when the heat flux relaxation time $\left(\tau_{\mathrm{q}}\right)$ become equal to the temperature response time $\left(\tau_{\mathrm{T}}\right)$, DPL model reduces to the PMBTE model. It was also concluded that as the radius of the incident heat flux spot increases tissue burn time decreases.

Heat transfer into skin tissue involves conduction through tissue, convection between tissue and blood, diffusion through micro vascular beds and metabolic heat generation. Lin and $\mathrm{Li}$ (2016) propose an analytical solution to the bio-heat transfer for skin tissues subjected to pulsed laser heating and fluid cooling. The analysis is performed using boundary conditions as proposed in the Penner, CattaneoVernotte(CV) and Dual-Phase Lag (DPL) models. The thermal damage of the tissue is modeled by the Arrhenius burn integration. The analysis shows that for large phase lag $\tau_{\mathrm{q}}$, the thermal wave speed is approximated as, $V_{T}=\sqrt{k / \tau_{q} p C}$.

Jasi'nski et al.(2016) numerically analyses a soft tissue subjected to laser irradiation using the DPL model in 3D domain. The internal heat generation due to laser irradiation and tissue destruction are taken into account. It was found that as tissue damage increases porosity is decreasing leading to loss of perfusion. This in turn affects the heat flux relaxation time $\left(\tau_{\mathrm{q}}\right)$, temperature response time $\left(\tau_{\mathrm{T}}\right)$, effective thermal conductivity and effective heat capacity.

Precise prediction and control of temperature is vital to the success of thermal therapy in treatment of metastatic cancerous cells. Kumar et al. (2015) has developed a space fractional hyperbolic bio-heat transfer model for living tissues. The fractional bioheat model shows that as phase lag time increases temperature at hyperthermia position decreases.

Kumar and Srivastava (2015) investigated the thermal response of biological tissues irradiated with laser using the Dual Phase Lag (DPL) model. The transient form of radiative transfer equation (RTE) is used to model the propagation of light through a biological tissue. Since the cancerous tissue has higher absorption coefficient, it results in localized temperature rise. The difference in temperature distributions due to the optical inhomogenities in biological tissues can be used for diagnosis as well as treatment.

Majchrzak and Turchan (2015) modeled the bioheating of 3D tissues using the dual phase lag equation which was solved using the general boundary element method. The modeling of biological tissues using the dual phase lag model helps to analyse the heterogeneous nature of the tissues.

Hooshmand et al.(2015) discusses an analytical solution for non-equillibrium heat transfer in biological tissues subjected to laser heating, modeled using the generalized dual phase lag model. The solution to the volume averaged local energy equation was arrived at using the separation of variables and Duhamel's integration methods.

Liu and Chen (2016) models the non-equillibrium effect due to heating of tissues using the general dual phase lag model. The thermal damage is quantified using the Arrhenius equation and the modified discretization technique and Laplace transform was applied in a hybrid form to solve the equation.

Ahmadikia and Moradi (2012) presented an analysis based on Non-Fourier model, of phase change heat transfer in biological tissues during a freezing process. The simulation was based on hyperbolic heat equations with temperature-dependent enthalpy, which were compared with the parabolic model. Numerical solutions were obtained using the Finite Difference method. The results indicated that the Fourier model under predicted temperatures, compared to the non-Fourier model.

Gowrishankar et al. (2004) investigated on bio-heat transfer in skin with spatially heterogeneous, temperature-dependent perfusion, using a transport lattice approach. The heat transport processes were modeled using a lattice that represents the Pennes bioheat equation in perfused tissues, and diffusion in non-perfused regions. The heat transport model of the skin was solved by utilizing an electrical analogy. Srivastava et al. (2009) has used a new homotopy perturbation method to solve the non-Fourier bioheat transport problem in microscale bio-films.

Mach et al. (2011) applied Lattice Boltzmann method to analyze the heat transfer in cereal-based foam. The heat transfer processes were modeled with a microstructural point of view. The complex thermo-fluidic processes pertained to the solid and gas phases, introduced the microscale dynamics, and used Lattice Boltzmann Method. The results quantified the effects of porosity and interconnectivity of gas pores in bread crumbs, on the overall heat transfer. 
A novel meshless radial basis collocation method (RBCM) was used by Jamil and $\mathrm{Ng}$ (2013) to analyze heterogeneous conduction and determine the temperature in biological tissues. RBCM was utilized to simulate the bioheat transfer problem in the two dimensional domain. The meshless nature, accuracy and point-based data dependency of the method makes it an attractive option for solving the bio-heat transfer problem.
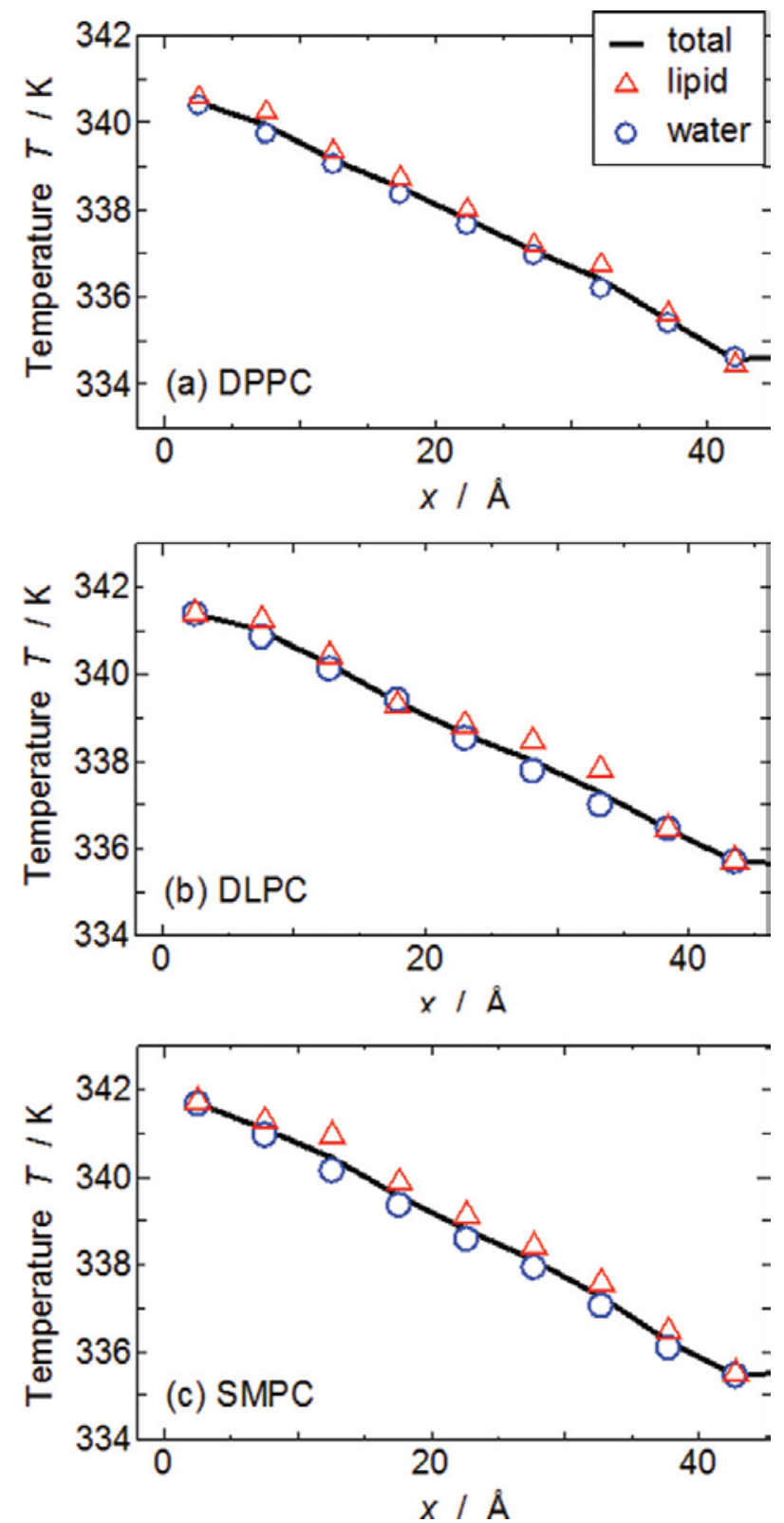

Figure 6. Temperature distributions along membranes in lipid systems of (a) DPPC, (b) DLPC, and (c) SMPC. From Nakano et al. (2013).

Bhowmik and Repaka (2016) employed genetic algorithm to analyse the sub surface cancer tissues from the temperature measurements on the skin surface. It was attempted to quantify the tumor diameter, penetration depth and heat generation from the temperature distribution on skin surface captured using a thermal camera. This helps in diagnosis of melanoma (sub surface skin cancer) without the need of biopsy as it is a non-invasive and non-contact technique. In the present work the change in surface thermal responses captured using thermal camera were modeled as a forward model and supplied to a data-mining inverse algorithm to arrive at the in-vivo features of the tumor. The analysis was done 
for Clark II and Clark IV classes of melanoma. It was found that simulated annealing (SA) algorithm was superior to genetic algorithm (GA) due to faster retrieval and reasonable accuracy.

Molecular Dynamics (MD) simulation has been utilized to analyze bio-heat transfer problems, with an atomistic perspective. Lin (2011) studied heat transfer phenomena in bio tissue subjected to external heating, using the approach. The simulation focused on the temperature evolution and thermal conductivity of Alanine molecules. The study is relevant to applications such as thermal ablation and micro-scale hyperthermia. Nakano et al. (2013) analyzed molecular heat transfer in lipid bilayers, using Non-Equilibrium Molecular Dynamics simulations. Simulations were conducted on dipalmitoylphosphatidyl-choline (DPPC), dilauroyl-phosphatidyl-choline (DLPC), and stearoyl-myristoylphosphatidyl-choline (SMPC). The results provided useful information on transport characteristics of thermal energy in biocompatible materials. Typical simulation results on temperature distributions are shown in Fig. 6.

\section{$5 \quad$ Experimental Investigations}

Publications reporting experimental studies, using conventional and special instrumentation techniques to visualize and measure thermal phenomena in biological systems have appeared in the literature in recent times. Some of these pertain to the microscale measurements domain. While some of the investigations were focused on understanding and analyzing the phenomena, others were aimed at addressing problems in specific applications, as well as developing methodologies for new products and practices. Some of the important publications are reviewed here.

Rubinsky (1997) discussed extensively on microscale heat transfer in biological systems at low temperatures, in his review paper. Thermal phenomena occurring at micro and nanoscale were discussed, dividing temperatures to those above and below freezing. Biomedical applications of low temperatures in cryopreservation of organs for transplantation and destruction of undesirable tissues by freezing in cryosurgery were also discussed, with electron micrographs pertinent to the understanding of the concepts.

Ricketts et al. (2008) have reported non-invasive blood-perfusion measurement using combined temperature and heat flux surface probes. The probes utilized a small sensor with a laminated flat thermocouple to measure the heat transfer and temperature response, and the blood perfusion and thermal contact resistance were estimated by comparing heat flux data with a mathematical model of the tissue, based on Pennes bio-heat equation. Different designs were discussed. The probes were found to be effective in detecting small changes in perfusion, as low as $0.005 \mathrm{ml}$, per $\mathrm{ml} / \mathrm{s}$ of blood flow.
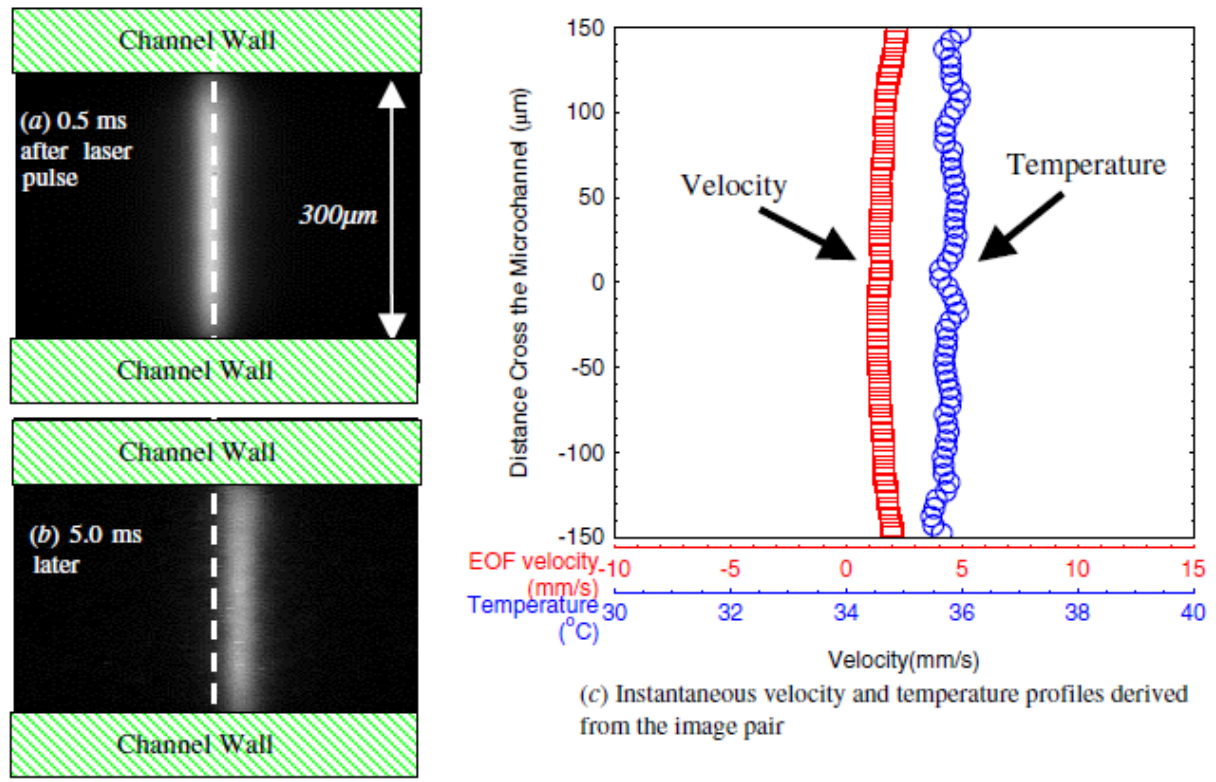

(c) Instantaneous velocity and temperature profiles derived from the image pair

Figure 7. Molecular tagging measurement in electrokinetically driven flow. From Hu et al. (2010) 
$\mathrm{Hu}$ et al. used molecular tagging technique for simultaneous measurement of velocity and temperature, using specially designed phosphorescent molecules, which produce glowing marks on excitation by photons of appropriate wavelength. Simultaneous velocity and temperature measurement were achieved by measuring the Lagrangian displacement of the tagged molecules, and utilizing the temperature dependence of the phosphorescence lifetime, respectively, utilizing two phosphorescence images. The method was demonstrated by making measurements in electro-osmotic flow inside a microchannel (as shown in Fig. 7), and also by measuring unsteady heat transfer and phase change inside micro-sized water droplets.

Chua (2013) presented experimental studies on cryo-freezing. Extensive experimental studies were conducted to validate theoretical models, incorporating the effect of introducing gold nanoparticles of 30 nanometers size, to enhance heat conduction and control freezing to minimize unintended cryo-injury to the neighboring tissues. Temperature profiles generated from the computation were compared with those measured in the in vitro studies. Thermocouple measurements and infrared thermography were utilized, with gelatin and animal liver as the experimental substances.

Thermophoresis is the directed motion of molecules in temperature gradients. Seidel et al. (2013) utilized microscale thermophoresis for quantitative analysis of protein interactions in free solution and with low sample consumption. The technique is based on an optical measurement approach, in which an infrared laser was used for local heating, and the molecule motion was analyzed using the fluorescence of one of the binding partners. Measurement was possible even in complex bio-liquids like cell lysate, allowing close to in vivo conditions without sample purification.

Roper et al. (2007) discussed in their publication, aspects of microscale heat transfer transduced by gold nanoparticles, as explained by surface plasmon resonance. In the experimental study, temperature in aqueous suspensions of 20-nm gold particles irradiated by a continuous wave argon- ion laser at 514 $\mathrm{nm}$ was increased to a maximum equilibrium value. This value increased was found to be in proportion to incident laser power and to the nanoparticle content at low concentration. The microscale heat transfer time constant was determined for the gold nanoparticle suspension, from the transient temperature profile. Thermocouple measurements were utilized in the study.

Thermochormic Liquid crystals are being used for thermal sensing in microfluidic devices. Organic compounds like cholesteryl esters, phenyl-4-alkyl benzotaes, etc. are the most popular thermochromic liquid crystals with structure falling between that of a crystalline and liquid. They have superposed planes with temperature dependent separation distances.TLCs encapsulated in polymeric materials are used by Gao et al. (2014) for thermography to map skin temperatures.

TLCs can also be used for controlling local temperatures to enhance biosensing. It can also be used to sense temperatures without disturbing the local thermal field as in the case of polymerase chain reaction biorecognition systems which are highly temperature dependent (Chaudhari et al., 1998).

Capture induced hyperthermia is considered as a major cause of morbidity and mortality of wild animals captured for translocation or research. Core body temperatures of wild animals are being monitored with microchip thermometry so that they can be cooled by water-dousing. Rey et al.(2016) conducted a study on springboks by inserting microchips subcutaneously and into gluteus muscles. The subcutaneous temperatures failed to reflect accurately the core body temperatures as compared to the muscle temperatures.

Heat stress has shown to cause adverse biological and physiological effects in human and animals. Investigations by Giblot Ducray et al. (2016) showed that yeast fermentate Epicor (EH) can prevent heat stress related complications in rat. The exposal of animals like rats to heat stress was found to increase the body temperature resulting in morphological changes in intestine. The effect of heat stress in reducing mucosal thickness was found to be mitigated by treatment with yeast fermentate Epicor (EH). The pretreatment with EH was also found to keep the white blood cell (WBC) concentration, free vescicles concentration and diameter in the blood unchanged when exposed to heat stress.

There have been interesting publications describing new methodologies and developing new devices for bio-engineering applications. Kleinstreuer et al. (2008) described a methodology for targeting drugaerosols in human respiratory system, in the context of inhalation of medicine. The new methodology is based on the use of a controlled air-particle stream, providing a patient-specific drug-aerosol deposition. Maximum deposition, based on optimal particle diameter and density, inhalation waveform, and particle-release position has been aimed at, which is essentially required during the use of targeted 
medicines. The methodology was suggested based on computational and experimental study, thus providing the optimal combination of the parameters.

Reviews and discussions on measurements on general and application-specific microfluidic systems are also found in the literature. Discussions on theoretical modelling, simulations and experiments pertaining to microfluidics applicable to lab-on-a-chip devices can be found in the article by Li (2005). Choi and Bischof (2010) reviewed measurement methods for material and thermal properties of biomaterials in the crvogenic regime, for prediction of freezing in crvobiologv applications. The review revealed a lack of information of properties for many biomaterials, especially for systems with cryo-protective agents, at subzero temperatures. Jain and Goodson (2011) have extensively discussed thermal microdevices which find applications in biological and bio-medical systems. The paper focused on microfabrication technology, with emphasis on biomedical applications. Applications of thermal devices and tools in the study of cellular thermal interactions and biological macromolecules were reviewed. The paper discussed MEMS based temperature measurement techniques, and bio-medical and bio-analytical devices and techniques. Miralles et al. (2013) has presented a review of the techniques and applications of heating and temperature control in microfluidic systems. Recent strategies where integration of a heating source to generate a temperature gradient, thus offering control of a key parameter, as well as methods where high accuracy temperature gradient focusing is utilized, are examined in this paper.

\section{Micro Heat Spreader Applications in Bio-Medical Systems}

The micro heat pipe is a passive heat transfer device, with extremely high effective thermal conductance. These essentially consist of arrays of polygonal cross section channels, the corners of which produce capillary action to circulate a working fluid that undergoes phase change, in order to transport heat (Sobhan and Peterson, 2008). The high effective thermal conductance and the compact size of micro heat pipes make them attractive as heat spreaders, which can spread localized heat fluxes over a larger surface area.

Suitable designs of the micro heat pipe heat spreader have been developed which are useful in interesting bio-medical applications. Fletcher and Peterson (1993) developed catheters which provide a hyperthermia or hypothermia source, effective in the treatment of tumors and cancers. In one of the designs, the heat pipe has the size of a hypodermic needle, and is thermally insulated along a substantial portion of its length. It has a channel, partially charged with an appropriate working fluid. The device provides the delivery or removal of thermal energy directly or from a tumor or diseased tissue site. In a second design, the catheter uses a variety of passive heat pipe structures alone or in combination with feedback devices. This catheter is particularly useful in treating diseased tissue that cannot be removed by surgery, such as a brain tumor.

Polymer-based micro heat pipe heat spreaders have been developed and tested, for treatment of neocortical seizures. These are designed as small heat spreaders which are implantable. The heat spreader extracts heat from hot-spots in the brain, and spreads it over a larger area, thus effectively providing localized cooling. Hilderbrand et al. (2007) have discussed the design, development and testing of such heat spreaders. In this work, an analytical and experimental investigation has been reported, leading to the development of flat phase change heat spreaders for local thermoelectric cooling as a treatment for intractable neocortical epilepsy. The use of polymer films with microchannels to produce wicking structure in the heat spreaders are also discussed in this paper. The structure of a polymer material useful for heat pipe heat spreaders for bio-medical application is shown in Fig.8.

Spinning off from conventional designs having fabricated microchannels, new designs of micro heat pipes with passages formed by wire-arrays sandwiched between plates (wire-bonded or wire sandwiched micro heat pipes) have been proposed (Wang and Peterson, 2002; Sobhan et al., 2007; Sobhan and Peterson, 2008). Attempts have been successfully made to design and fabricate wire-sandwiched heat pipes for bio-medical applications in external hyperthermia of the body. In a recent effort, wire sandwiched micro heat pipes have been fabricated using flexible PDMS sheets, and tested at operating conditions suitable for external application in hyperthermia. Fig. 9 shows a photograph of a typical device of this kind. The micro heat pipe passages provide capillary flow with phase change, transmitting heat from the evaporator section, maintaining an almost uniform temperature at a required level. The line for working fluid charging is also seen in the photograph. Investigations are underway for optimizing the design parameters and determining the best operating conditions for this device. 


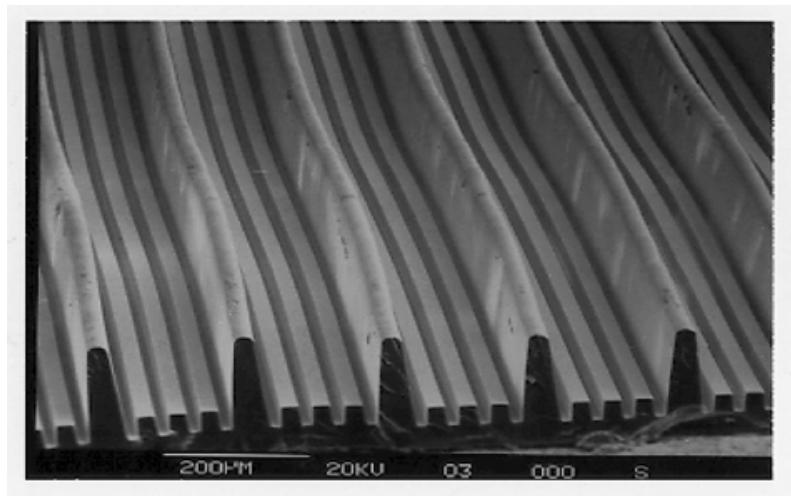

Figure 8. Polymer film material for micro heat pipe heat spreaders.

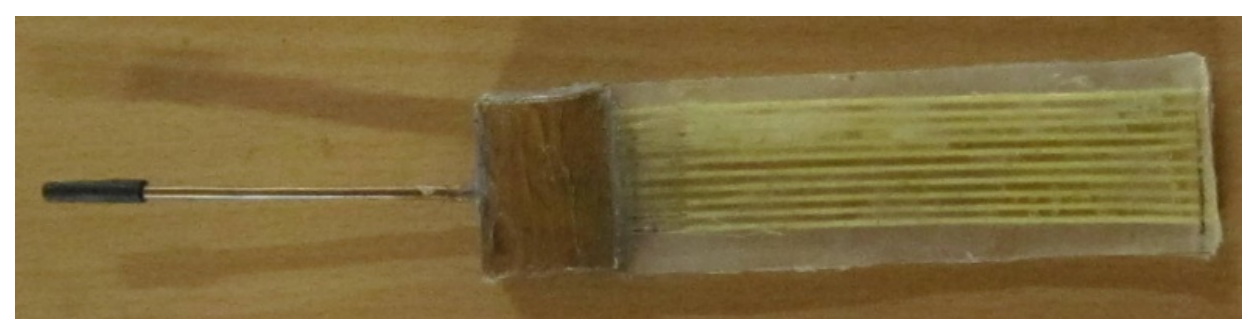

Figure 9. Wire-sandwiched polymer micro heat pipe designed for external heating

\section{Conclusion}

Investigations on microscale thermal energy transport phenomena related to biological systems and biomedical applications, reported in recent times, have been reviewed in this paper. The major areas of interest for study and analysis of thermal transport problems have been biological tissues, cryopreservation and mass transport in cell membranes. Some of the investigations were focused on particulate transport in biological systems also. Prediction of temperature fields and velocity distributions in blood-perfused tissues, with conventional and modified bio-heat transfer equations have been reported in various papers. Accurate prediction of the temperature field and extend of damage to the tissue is important in thermal therapy using focused laser beam. The Fourier's law breaks down in the case of heating in biological tissues owing to their non-homogeneous material structure and high power with short durations, occasionally subjected to cryogenic temperatures. Owing to the finite speed of thermal wave propagation in biological tissues, Dual Phase Lagging model for heat conduction is the preferred model off late. The vascular network in the tissues is being modeled accurately with the help of MRI images. Most of the theoretical investigations utilized numerical methods for solution of the relevant governing equations, while there have been a few papers on discrete computations and molecular simulations. There has been considerable amount of studies reported on laser heating of biological tissues. Experimental research using simulated as well as original biological tissues have been reported. Investigations are also proceeding in the direction of predicting damages to tissues by experimentally measuring the temperature distribution on skin surface using thermal imaging. The temperature distributions obtained from theoretical analysis of damaged tissues are being correlated with the experimental measurements to make the prediction more accurate. Materials like thermochromic liquid crystals are being used in thermography to measure temperature distributions in biological tissues accurately. Research focused on development of heat transport devices such as heat spreaders for biological applications also have been reviewed. 


\section{References}

1. Ahmadikia, H. and Moradi, A., "Non-Fourier phase change heat transfer in biological tissues during solidification", Heat and Mass Transfer, 48, pp. 1559-1568, 2012.

2. Arkin, H., Xu, L. X. and Holmes, K. R., "Recent developments in modeling heat transfer in blood perfused tissues", IEEE Transactions on Biomedical Engineering, 41(2), pp. 97-107, 1994.

3. Askarizadeh, H. and Ahmadikia, H., "Analytical study on the transient heating of a two-dimensional skin tissue using parabolic and hyperbolic bioheat transfer equations", Applied Mathematical Modelling, 39, pp. 3704-3720, 2015 .

4. Banerjee, A., Ogale, A. A., Das, C., Mitra, K. and Subramanian C., "Temperature distribution in different materials due to short pulse laser irradiation", Heat Transfer Engineering, Vol. 26, No 8, pp. 41-49, 2005.

5. Bhowmik, A. and Repaka, R., "Estimation of growth features and thermophysical properties of melanoma within 3-D human skin using genetic algorithm and simulated annealing", International Journal of Heat and Mass Transfer, 98, pp. 81-95, 2016.

6. Bischof, J. C. and Rubinsky, R., "Microscale heat and mass transfer of vascular and intracellular freezing in the liver", ASME J Heat Transfer, 115, pp. 1029-1035, 1993.

7. Chaudhari, A.M., Woudenberg, T.M., Allin, M., Goodson, K.E., "Transient liquid crystal thermometry of microfabricated PCR vessel arrays", Journal of Microelectromech. Syst., 7, pp. 345, 1998.

8. Chen, H., Shen, H., Heimfeld, S., Tran, K. K., Reems, J., Folch, A. and Gao, D., "A mirofluidic study of mouse dendritic cell membrane transport properties of water and cryoprotectants", Int J Heat Mass Transfer, 51, pp. 5687-5694, 2008

9. Choi, J. and Bischof, J. C., "Review of biomaterial thermal property measuements in the cryogenic regime and their use for prediction of equilibrium and non-equilibrium freezing applications in cryobiology", Cryobiology, 60(1), pp. 52-70, 2010.

10. Chua, K. J., "Fundamental experiments and numerical investigations of cryo-freezing incorporating vascular network with enhanced nano-freezing", Int J Thermal Sciences, 70, pp. 17-31, 2013.

11. Davalos, R. V. and Rubinsky, B., "Temperature considerations during irreversible electroporation", Int J Heat Mass Transfer, 51, pp. 5617-5622, 2008.

12. Devireddy, R. V., Smith, D. J. and Bischof, J. C., "Effects of microscale mass transport and phase change on numerical prediction of freezing in biological tissues", ASME J Heat Transfer, 124, pp. 365-374, 2002.

13. Eshpuniyani, B., Fowlkes, B. and Bull, J. L., "A boundary element model of microbubble sticking and sliding in the microcirculation", Int J Heat Mass Transfer, 51, pp. 5700-5711, 2008.

14. Fleming Glass, K. K., Longmire, E. K. and Hubel, A., "Optimization of a microfluidic device for diffusion-based extraction of DMSO from a cell suspension", Int J Heat Mass Transfer, 51, pp. 5749-5757, 2008.

15. Gao, L., Zhang, Y., Malyarchuk, V., Jia, L., Jang, K.-I., Chad Webb, R., Fu, H., Shi, Y., Zhou, G., Shi, L., Shah, D., Huang, X., Xu, B., Yu, C., Huang, Y. and Rogers, J.A., "Epidermal photonic devices for quantitative imaging of temperature and thermal transport characteristics of the skin", Nat. Commun., 5, pp. 4938, 2014.

16. Giblot Ducray, H.A., Globa, L., Pustovyy, O., Reeves, S., Robinson, L., Vodyanoy, V. and Sorokulova, I., "Mitigation of heat stress-related complications by a yeast fermentate product", Journal of Thermal Biology, 60, pp. 26-32, 2016.

17. Gowrishankar, T. R., Stewartm D. A., Martin, G. T. and Weaver, J. C., "Transport lattice models of heat transport in skin with spatially heterogeneous, temperature dependent perfusion", Biomedical Engineering Online, 3, pp. 42, 2004, doi:10.1186/1475-925X-3-42.

18. Grabski, J., Lesnic, D. and Johansson, B.T., "Identification of a time-dependent bio-heat blood perfusion coefficient", International Communications in Heat and Mass Transfer, 75, pp. 218-222, 2016.

19. Granot, Y. and Rubinsky, B., "Mass transfer model for drug delivery in tissue cells with reversible electroporation", Int J Heat Mass Transfer, 51, pp. 5610-5616, 2008.

20. Guan, K. W., Jiang, Y. Q., Sun, C. S. and H. Yu, "A two-layer model of laser interaction with skin, A photothermal effect analysis", Optics \& Laser Technology, Vol 43, pp 425-429, 2011.

21. Heussner, N., Holl, L., Nowak, T., Beuth, T., Spitzer, M.S. and Stork, W., "Prediction of temperature and damage in an irradiated human eye-Utilization of a detailed computer model which includes a vectorial blood stream in the choroid", Computers in Biology and Medicine, 51, pp. 35-43, 2014. 
22. Hilderbrand, J. K., Peterson, G. P., Rothman, S. M., "Development of phase change heat spreader for treatment of intractable neocortical epilepsy", Heat Transfer Engineering, 28, 4, pp. 282-291, 2007.

23. Hooshmand, P., Moradi, A. and Khezry, B., "Bioheat transfer analysis of biological tissues induced by laser irradiation", International Journal of Thermal Sciences, 90, pp. 214-223, 2015.

24. Hu, H., Jin, Z., Nocera, D., Lum, C. and Koochesfahani, M., "Experimental investigations of micro-scale flow and heat transfer phenomena by using molecular tagging techniques", Measurement Science and Technology, 21, pp. 085401 (14 pp.), 2010. doi:10.1088/0957-0233/21/8/085401

25. Jain, A. and Goodson, K. E., "Thermal microdevices for biological and biomedical applications", Journal of Thermal Biology, 36, pp. 209-218, 2011.

26. Jamil, M. and Ng, E. Y. K., "Evaluation of meshless radial basis collocation method (RBCM) for heterogeneous conduction and simulation of temperature inside the biological tissues", Int J Thermal Sciences, 68, pp. 42-52, 2013.

27. Jasi'nski. M., Majchrzak, E. and Turchan, L., "Numerical analysis of the interactions between laser and soft tissues using generalized dual-phase lag equation", Applied Mathematical Modelling, 40, pp. 750-762, 2016.

28. Jaunich, M., Raje, S., Kim, K., Mitra, K and Guo, Z., "Bio-heat transfer analysis during short pulse laser irradiation of tissues", Int. J. Heat Mass Transfer, Vol.51, pp 5511-5521, June 2008.

29. Jiao, J. and Z. Guo, Z., "Thermal interaction of short-pulsed laser focused beams with skin tissues", Phys. Med. Biol., Vol. 54, pp 4225-4241, June 2009.

30. Kandra, D. and Devireddy, R. V., "Numerical simulation of local temperature distortions durng ice nucleation of cells in suspension", Int J Heat Mass Transfer, 51, pp. 5655-5661, 2008.

31. Kappiyoor, R., Liangruksa, M., Ganguly, R. and Puri, I. K., "The effects of magnetic nanoparticle properties on magnetic fluid hyperthermia", Journal of Applied Physics, 108, pp. 094702:1-8, 2010.

32. Kathawate, J. and Acharya, S., "Computational modeling of intravitreal drug delivery in the vitreous chamber with different vitreous substitutes", Int J Heat Mass Transfer, 51, pp. 5598-5609, 2008.

33. Katsidis, C. C., "A simple model for the analysis of light absorption and temperature rise in human skin: the role of surface roughness", Department of Applied Informatics and Multimedia, A.T.E.I of Crete, Heraklion, Greece, 2002.

34. Kim, K. and Guo, Z., "Multi-time-scale heat transfer modeling of turbid tissues exposed to short-pulsed irradiations", Comp. Meth. Prog. Biomed., Vol. 86, pp 112-123, 2007.

35. Kleinstreuer, C., Li, J. and Koo, J., "Microfluidics of nano-drug delivery", Int J Heat Mass Transfer, 51, pp. 5590-5597, 2008.

36. Kleinstreuer, C., Zhang, Z., Li, Z., Roberts, W. L. and Rojas, C., "A new methodology for targeting drugaerosols in the human respiratory system", Int J Heat Mass Transfer, 51, pp. 5578-5589, 2008.

37. Kumar, P., Kumar, D. and Rai, K.N., "A mathematical model for hyperbolic space-fractional bioheat transfer during thermal therapy", Procedia Engineering, 127, pp. 56 - 62, 2015.

38. Kumar, S. and Srivastava, A., "Thermal analysis of laser-irradiated tissue phantoms using dual phase lag model coupled with transient radiative transfer equation", International Journal of Heat and Mass Transfer, 90, pp. 466-479, 2015.

39. Kuznetsov, A. V. and Hooman, K., "Modeling traffic jams in intracellular transport in axons", Int J Heat Mass Transfer, 51, pp. 5695-5699, 2008.

40. Lee, Y.W., "Novel Design of integrated microfluidic thermal system with self-assembling magnetic particles for cooling", Microelectronic Engineering, 111, pp. 285-288, 2013.

41. Li, D., "Microfluidics in lab-on-a-chip: Models, simulations and experiments", in Kakac et al. (eds.), Microscale Heat Transfer, Springer, Netherlands, pp. 157-174, 2005.

42. Liangruksa, M., Ganguly, R. and Puri, I. K., "Parametric investigation of heating due to magnetic fluid hyperthermia in a tumor with blood perfusion", Journal of Magnetism and Magnetic Materials, 323, pp. 708-716, 2011.

43. Lin, D. T. W., "A molecular dynamics study of the heat transfer phenomena in the bio-tissue", Int J Science and Engineering, 1(2), pp. 17-21, 2011.

44. Lin, S.-M. and Li, C.-Y., "Analytical solutions of non-Fourier bio-heat conductions for skin subjected to pulsed laser heating", International Journal of Thermal Sciences, 110, pp. 146-158, 2016. 
45. Liu, J., "Preliminary survey on the mechanisms of the wave-like behaviors of heat transfer in living tissues", Forschung im Ingenieurwesen, 66, pp. 1-10, 2000.

46. Liu, K.-C. and Chen, Y.-S., "Analysis of heat transfer and burn damage in a laser irradiated living tissue with the generalized dual-phase-lag model", International Journal of Thermal Sciences, 103, pp. 1-9, 2016.

47. Ma, W., Liu, W. and Li, M., "Analytical heat transfer model for targeted brain hypothermia", International Journal of Thermal Sciences, 100, pp. 66-74, 2016.

48. Mack, S., Hussein, A. and Becker, T., "Investigation of heat transfer in cereal-based foam from a micro-scale perspective using the lattice Boltzmann method", J. Non-Equib. Thermodyn., 36, pp. 311-335, 2011.

49. Majchrzak, E. and Turchan, L., "The general boundary element method for 3D dual-phase lag model of bioheat transfer", Engineering Analysis with Boundary Elements, 50, pp. 76-82, 2015.

50. Merrikh, A. A. and Lage, J. L., "Plasma microcirculation in alveolar capillaries: Effect of parachute-shaped red cells on gas exchange", Int J Heat Mass Transfer, 51, pp. 5712-5720, 2008.

51. Miralles, V., Huerre, A., Malloggi, F. and Jullien, M. C., "A review of heating and temperature control in microfluidic systems: Techniques and applications", Diagnostics, 3, pp. 33-67, 2013. doi: 10.3390/diagnostics3010033.

52. Molina, J., Rivera, M. and Trujillo, M., "Assessment of hyperbolic heat transfer equation in theoretical modeling for radiofrequency heating techniques", The open Biomedical engineering journal, Vol. 2, pp 22-27, 2008.

53. Nakano, T., Kikugawa, G. and Ohara, T., "Molecular heat transfer in lipid bilayers with symmetric and asymmetric tail chains", ASME J Heat Transfer, 135, pp. 061301:1-8, 2013.

54. Norouzi, M., Davoodi, M., Beg, O, A. and Joneidi, A. A., "Analysis of the effect of normal stress differences on heat transfer in creeping viscoelastic Dean flow", International Journal of Thermal Sciences, 69, pp. 61-69, 2013.

55. Özen, S., Helhel, S. and Bilgin, S., "Temperature and burn injury prediction of human skin exposed to microwaves: a model analysis", Radiation and Environmental Biophysics, Vol. 50, No 3, pp 483-489, April 2011.

56. Pennes, H.H., "Analysis of tissue and arterial blood temperatures in the resting human forearm", J. Appl. Physiol., 1, pp. 93-122, 1948.

57. Rey, B., Fuller, A., Hetem, R.S., Lease, H.M., Mitchell, D. and Meyer, L.C.R., "Microchip transponder thermometry for monitoring core body temperature of antelope during capture", Journal of Thermal Biology, 55, pp. 47-53, 2016.

58. Ricketts, P. L., Mudaliar, A. V., Ellis, B. E., Pullins, C. A., Meyers, L. A., Lanz, O. I., Scott, E. P. and Thomas E. Diller, T. E., "Non-invasive blood perfusion measurements using a combined temperature and heat flux surface probe", Int J Heat Mass Transfer, 51, pp. 5562-5577, 2008.

59. Roper, D. K., Ahn, W. and Hoepfner, M., "Microscale heat transfer transduced by surface plasmon resonant gold nanoparticles", J. Phys. Chem. C, 111, pp. 3636-3641, 2007.

60. Rothman, S. M., Smyth, M. D., Yang, X. F., Peterson, G. P., "Focal cooling for epilepsy: An alternative therapy that might actually work", Epilepsy and Behavior, 7, pp. 214-221, 2005.

61. Rubinsky, B., "Microscale heat transfer in biological systems at low temperatures", Experimental Heat Transfer, 10, pp. 1-29, 1997.

62. Sakurai, A., Nitta, I., Maruyama, S., Okajima, J and Matsubara, K., "Coupled photon and heat transport simulation inside biological tissue for laser therapy", J. Thermal Science and Technology, Vol. 4, No 2, pp 314323, July 2009.

63. Santos, I., Haemmerich, D., Pinheiro, C. , Ferreira da Rocha, A., "Effect of variable heat transfer coefficient on tissue temperature next to a large vessel during radiofrequency tumor ablation", Biomedical Engineering Online, 7, 21, 2008. doi: 10.1186/1475-925X-7-21.

64. Sarkar, D., Haji-Sheikh, A. and Jain, A., "Temperature distribution in multi-layer skin tissue in presence of a tumor", International Journal of Heat and Mass Transfer, 91, pp. 602-610, 2015.

65. Seidel, S.A.E. et al. (17 authors), "Microscale thermophoresis quantifies biomolecular interactions under previously challenging conditions", Methods, 59, pp. 301-315, 2013.

66. Sinha, A. and Shit, G.C., "Electromagnetohydrodynamic flow of blood and heat transfer in a capillary with thermal radiation", Journal of Magnetism and Magnetic Materials, 378, pp. 143-151, 2015.

67. Sobhan, C. and Garimella, S,V., "A Comparative Analysis of Studies on Heat Transfer and Fluid Flow in Microchannels", Microscale Thermophysical Engineering, Vol. 5, pp. 291-309, 2001. 
68. Sobhan, C. B. and Peterson, G. P., "Microscale and Nanoscale Heat Transfer - Fundamentals and Engineering Applications". CRC Press/Taylor and Francis, 2008.

69. Sobhan, C. B., Rag, R. L., and Peterson, G. P., "A review and comparative study of the investigations on micro heat pipes", International Journal of Energy Research, 31, pp. 664-688, 2007.

70. Srivastava, V., Rai, K.N. and Das, S., "Analytical approach to micro scale bio-film heat transport using homotopy perturbation method", Int J Applied Mathematics and Computation, 1, 3, pp. 148-158, (2009). (http://ijame.darbose.com).

71. Tzou, D.Y., "A unified field approach for heat conduction from macro- to microscales", ASME Journal of Heat Transfer, Vol. 117, pp 8-16, 1995.

72. Vu, H., García-Valladares, O. and Aguilar, G., "Vapor/liquid phase interaction in flare flashing sprays used in dermatologic cooling", Int J Heat Mass Transfer, 51, pp. 5721-5731, 2008.

73. Wang, K., Tavakkoli, F., Wang, S. and Vafai, K., "Analysis and analytical characterization of bioheat transfer during radiofrequency ablation", Journal of Biomechanics, 48, pp. 930-940, 2015.

74. Wang, Y. X. and Peterson, G. P., "Analysis of Wire-Bonded Micro Heat Pipe Arrays", Journal of Thermophysics and Heat Transfer, Vol. 16, No. 3, pp. 346-355, 2002.

75. Wang, Z., Zhao, G., Wang, T., Yu, Q., Su, M. and He, X., "Three-dimensional numerical simulation of the effects of fractal vascular trees on tissue temperature and intracellular ice formation during combined cancer therapy of cryosurgery and hyperthermia", Applied Thermal Engineering, 90, pp. 296-304, 2015.

76. Wessapan, T. and Rattanadecho, P., "Specific absorption rate and temperature increase in the human eye due to electromagnetic fields exposure at different frequencies", International Journal of Heat and Mass Transfer, 64, pp. 426-435, 2013.

77. Xi, J. and Longest, P. W., "Numerical predictions of submicrometer aerosol deposition in the nasal cavity using a novel drift flux approach", Int J Heat Mass Transfer, 51, pp. 5562-5577, 2008.

78. Xu, F, Moon, S., Zhang, X., Shao, L., Song, Y.S. and Demirci, U., "Multi-scale heat and mass transfer modelling of cell and tissue cryopreservation", Phil. Trans. R. Soc. A, 368, 2010. doi: 10.1098/rsta.2009.0248.

79. Zhang, J. Z., Shen, Y. G. and Zhang, X. X., "A dynamic photo-thermal model of carbon dioxide laser tissue ablation", Lasers Med Sci, Vol. 24, pp 329-338, June2008.

80. Zhang, Y., "Generalized dual phase lag bioheat equations based on nonequilibrium heat transfer in living biological tissues", Int J Heat Mass Transfer, 52, pp. 4829-4834, 2009.

81. Zhou, J., Chen, J. K. and Zhang, Y., "Dual-phase-lag effects on thermal damage to biological tissues cause by laser irradiation", Computer in Biology and Medicine, Vol. 39, pp 286-293, January2009.

82. Zhou, J., Zhang, Y. and Chen, J.K., "An axisymmetric dual phase-lag bioheat model for laser heating of living tissues", Int. J. Thermal science, Vol. 48, pp 1477-1485, January 2009. 